\title{
A COMBINED OPHTHALMOSCOPE, RETINOSCOPE, AND HAND SLIT-LAMP
}

BY

\section{Stenhouse Stewart}

HULL

THE optical system of the instrument comprises a straight spiral filament (the source of illumination), a double plano-convex condenser, modifying slides, a focusing lens, and a mirror, with a supplementary lens and alternative mirror for special purposes.

As an ophthalmoscope, the light from the filament is rendered parallel by the condenser and falls upon a platform which carries two channels to receive the modifying slides. The largest aperture available selects a parallel pencil of rays smaller in diameter than the narrower tube which it then enters; in this way the tube reflections have been eliminated. The focusing lens is to be placed at its focal distance from the platform, a position indicated by a mark on the instrument. The filament is now brought to a focus at the mouth of the tube below the mirror, and the rays from the modifying slide made parallel. The optical system is slightly decentred, so that the beam falls just off the aperture in the mirror silvering, and as it has only just passed its focus, it is small enough to do this without loss.

In an emmetropic eye, the parallel rays from the platform are focused on the fundus as a sharp circle, while the virtual source of illumination (the filament image) is at or near the anterior focal point of the eye. This makes it impossible for the patient to focus the filament on his retina and prevents him from being dazzled, in spite of the luminosity employed. The sharp circle on the fundus is lit evenly by a beam almost parallel, and slight opacities in the media offer surprisingly little hindrance. In ametropia a movement of the focusing lens suitably adapts the beam.

The "corneal reflex" is weak and easily avoided; there are several reasons for this. In the pupillary area the reflected image is formed by the parallel rays from the slide which is a hole, and hence causes no interference whatever. The filament imagethe virtual source of light-is so near the cornea that its reflex is situated immediately behind the posterior corneal surface where it is easily avoided by parallax, and the reflected rays are so divergent that only a few pass through the sight hole to the observer's eye. Equally, the rays from the virtual source are highly divergent, only a few entering the mirror hole : no haze is noticeable even when the instrument is in use as a retinoscope.

For retinoscopy, a one millimetre hole-a virtual point sourceor a half-millimetre slit is used in conjunction with a ground glass on which the light from the bulb is evenly concentrated to provide 
a single secondary source. With the focusing lens in its primary position a parallel beam results; if the lens is moved down or up the beam becomes divergent or convergent to any extent desired. A rough judgment may be first obtained in high errors and a very fine estimation as the trial lens approaches the correct value. With the slit, one meridian is corrected at a time.

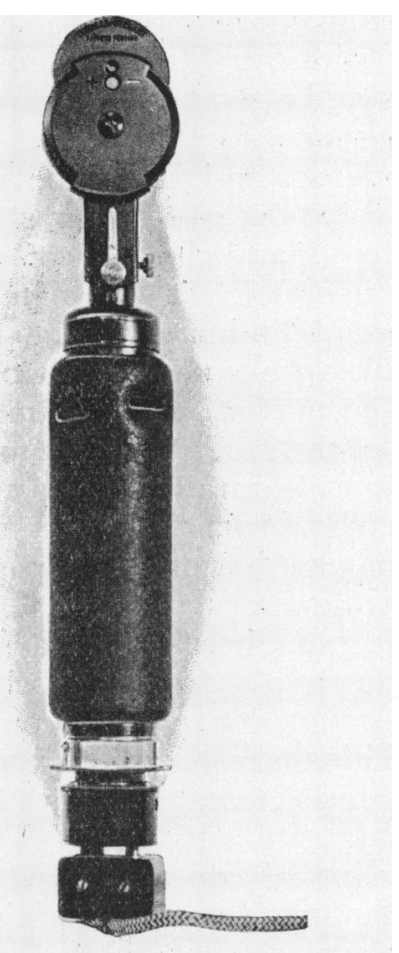

When the instrument is required for slit-lamp purposes, the focusing lens is set to the primary position which renders parallel the rays from the slit to be inserted in the platform channel. The top lens is swung into use and brings these rays to a slit focus at five centimetres distance; homogeneity is assured by the distance between the two lenses and the focusing of the filament image at the optical centre of the lower one; from this image parallel rays emerge from the top lens, so the setting is easily made by producing a sharp filament picture on a wall. The substitution of steel for glass mirror and other minor adjustments enable the slitbeam to be examined by the plus $20 \mathrm{D}$. lens in the instrument head.

*This instrument may be obtained from the makers, Messrs. Geo. Spiller, Ltd., 32, Wigmore Street, London, W.1. 
The Wratten $61 \mathrm{~N}$ red-free filters are employed in the usual way. At the suggestion of Dr. Margaret Dobson a special chromium screen is incorporated which utilises the foveal pigment to enhance the detail of the macular area. Any individual preferences in filters can be added quite simply, and the manufacturers will gladly give their assistance in any experimental work, scope for which is offered by the multiplicity of adjustments available.

\title{
EXTRACTION OF THORN FROM THE ORBITAL CAVITY
}

\author{
BY \\ S. N. KAUL, M.B., Ch.B.Edin. \\ LAHORE
}

BHAG Din, 35 years of age, applied for help in Sir Ganga Ram Free Hospital, Lahore, August 6, 1927.

History. While hedging four months ago, a foreign body entered his right eye behind the upper lid in its inner third. He cannot say what kind of foreign body it was, but all he can say is that it entered like a shot. Since then nothing has been done in the way of treatment.

On examination there is slight oedema of the right upper lid, chemosis of conjunctiva, and slight proptosis of the right eyeball, with a pin point pupil which does not react to light and accommodation. Anterior chamber is deep. Fundus is not visible and the pupil does not dilate with atropine. There is no tenderness on palpating the eyeball, and the right upper lid cannot be everted, and no foreign body can be seen, but, with gentle palpation, some hardness can be felt about the inner third of the lid. R.V.= P.L.; $\mathrm{L} . \mathrm{V} .=6 / 8$.

Under chloroform anaesthesia on the same day, when the lid was everted, a blackish mass was seen to be projecting out from the region of the fornix. With a little manipulation of the toothed conjunctival forceps, this was taken out and proved to be a thorn, one and a quarter inches in length. No pus escaped on pressure. The thorn was exactly like the one shown by Dr. Shroff of Bombay, and the site where it entered the fornix also corresponded to the picture shown by him in the February, 1928, number of the Brit. Jl. of Ophthalmology.

August 12,192\%. Very clean and clear eye, very little discharge behind the lid. A couple of granulations are present; these were snipped off. Pupil is slightly dilated and shows some exudate in the lower half, but fundus reflex is still unobtainable. There is no pain in the eye and never has been since the day of the operation. 